REVESCO. Revista de Estudios Cooperativos

ISSN: $1885-8031$

\title{
Las monedas complementarias y sociales en México
}

\author{
Karla Anel Andrews Serrano ${ }^{1}$ y Graciela Lara Gómez ${ }^{2}$ \\ Recibido: 10 de febrero de 2021 / Aceptado: 28 de diciembre de 2021 / Publicado: 26 de enero de 2022
}

Resumen. El dinero es un instrumento social de intercambio, que ha modificado su forma y denominación a través del tiempo. Su emisión es responsabilidad de la sociedad que lo reconoce y acepta como medio de pago. Actualmente, pueden identificar dos tipos de dinero: el primero se refiere al dinero mercancía el que es un medio de pago en especie y el segundo es el dinero fiduciario, que se basa en la fe o confianza de las comunidades, estados o países. Debido a las problemáticas derivadas de las crisis económicas, surgen las monedas complementarias y sociales como una iniciativa de personas que persiguen el beneficio común, significando una opción para reactivar y proteger la economía, cabe mencionar que con la creación de estas monedas no se pretende sustituir al dinero emitido por los países. Este tipo de monedas tiene un enfoque centrado en la confianza y en los lazos que existen entre la comunidad, no son reconocidas por el Estado, a pesar de ello se encuentran vigentes. De manera que, el objetivo de este estudio fue analizar e identificar las monedas complementarias y sociales emergentes en México. Para tal efecto, se recurrió al método documental, con el que se analizaron distintos tipos de monedas surgidas en contextos locales y que se encuentran en uso, tales como: créditos de servicio, mercados de intercambio mutuo, monedas locales y mercados de trueque. Los resultados muestran que el uso de monedas complementarias y sociales en México se ha difundido entre comunidades con fuertes carencias, siendo una alternativa viable para impulsar la actividad económica.

Palabras clave: Monedas sociales y complementarias; Créditos de servicio; Mercados de intercambio mutuo; Monedas locales; Mercados de trueque.

Claves Econlit: A13; P32; I131.

\section{[en] Complementary and social currencies in Mexico}

Abstract. Money is a social instrument of exchange, which has modified its form and denomination through time. Its issuance is the responsibility of the society that recognizes and accepts it as a means of payment. Currently, two types of money can be identified: the first refers to commodity money, which is a means of payment in kind and the second is fiduciary money, which is based on the faith or trust of communities, states, or countries. Due to the problems derived from the economic crises, complementary and social currencies arise as an initiative of people who pursue the common benefit, meaning an option to reactivate and support the economy, it is worth mentioning the creation of these currencies is not intended to replace the money issued by the countries. These types of currencies support an approach focused on trust and on the bonds that exist between the community, they are unrecognized by the State, despite this, they are still in force. Therefore, the objective of this study was to analyze and identify the emerging complementary and social currencies in Mexico. For this purpose, the documentary method was employed to analyze diverse types of currencies that have emerged in local contexts and are in use, such as service credits, mutual exchange markets, local currencies, and barter markets. The results show that the use of complementary and social currencies in Mexico has spread among communities with strong shortages, remains a viable alternative to increase economic activity.

Keywords: Social currency; Complementary currency; Service credits; Mutual exchange markets; Local currencies; Barter markets.

Sumario. 1. Introducción. 2. Objetivo y metodología. 3. Aproximación teórica a las monedas complementarias y sociales. 4. Resultados: las monedas complementarias y sociales en las comunidades mexicanas. 5. Discusión. 6. Conclusión. 7. Referencias bibliográficas.

Cómo citar. Andrews Serrano, K.A; Lara Gómez, G. (2022) Las monedas complementarias y sociales en México. REVESCO. Revista de Estudios Cooperativos, vol. 140, e79939. https://dx.doi.org/10.5209/reve.79939.

1 Universidad Autónoma de Querétaro, México.

Dirección de correo electrónico: anelandrews31@gmail.com.

2 Universidad Autónoma de Querétaro, México.

Dirección de correo electrónico: glara@ uaq.mx. 


\section{Introducción}

El dinero surge con el origen de la humanidad (Daly, 2008) es decir las sociedades antiguas utilizaban objetos que representaban cierta validez, eran escasos y se reconocían como medio de pago, por ejemplo: las semillas de cacao, sal, cigarros, conchas de mar, obsidiana y metales preciosos (Schettino, 2015; Graue, 2009). Con el tiempo, el factor dinero cambio de forma y denominación, persistiendo dos tipos: el primero, denominado como dinero mercancía, el cual vale por sí mismo o por su condición (como el oro o la plata); el segundo tipo se conoce como dinero convencional o fiduciario (Graue, 2009), que es decretado por los gobiernos de cada país para que sea legalmente aceptado, el cual funciona como medio de cambio (Mishkin, 2014).

De acuerdo con Lietaer (2001), el dinero fiduciario es un motor oculto del crecimiento de las sociedades industriales, primordialmente en el sistema económico capitalista. Para Hirota (2013) el sistema monetario predominante ha presentado sucesos que afectan a la mayoría de la población como la elevación de precios de bienes y servicios, pagos de interés compuesto y el enriquecimiento de la elite, provocando el empobreciendo debido al poco acceso a los mercados económicos y financieros. Por lo que, cuando existe una distribución concentrada del dinero y no alcanza a llegar a los sectores más vulnerables, se genera pobreza, desigualdad y marginación; es entonces que, para resolver parcialmente dichos problemas, surgen las monedas complementarias y sociales (Chaparro, 2014).

Las monedas complementarias y sociales representan un sistema monetario ad hoc que complementa y trabaja paralelamente con el sistema monetario oficial, sin pretender sustituirlo (Blanc, 2002), pero cubriendo necesidades que no son atendidas desde el sistema monetario convencional (Hirota, 2016). Corrons (2017) sugiere la denominación 'monedas complementarias', pero existen otras acepciones tales como: sociales, regionales, locales, comunitarias o alternativas. Este sistema complementario, es reconocido como paraeconómico o informal y se origina principalmente en las acciones de desarrollo local (Gisbert, 2010). Por tanto, las monedas sociales y complementarias son delimitadas por el espacio geográfico donde se empleen y sean aceptadas como medio de pago entre un grupo de personas y/o empresas (Orzi, 2007).

Con el artículo se lleva a cabo un acercamiento de corte documental basado en las principales monedas complementarias y sociales que han surgido en espacios locales en México. De manera que, se identifican tales monedas atendiendo a la clasificación de Seyfang y Longhurst (2013) como sigue: créditos de servicio, esquemas de intercambio mutuo, monedas locales y mercados de trueque. Luego se agrega un apartado de discusión y se cierra con las conclusiones.

Debe mencionarse que el uso de monedas complementarias y sociales ha tenido y tendrá auge nacional y mundial en las economías y han sido una alternativa en las crisis económicas recurrentes a lo largo de la historia, por lo que con ellas se busca suplir necesidades y buscar alternativas adecuadas para la población con mayores necesidades.

\section{Objetivo y metodología}

El objetivo del presente artículo fue identificar las principales monedas complementarias y sociales que han surgido en México durante el periodo de 1990 al 2020. El método de investigación empleado fue el documental, el cual presenta, describe e identifica el funcionamiento, uso, características, ventajas y contribuciones de tales medios de intercambio; los que han surgido en comunidades mexicanas y que se encuentran vigentes. Las fuentes de información consultadas se refieren a evidencias obtenidas de portales en internet, donde se presentan evidencias de la introducción y uso de monedas sociales y complementarias en las comunidades. Para el análisis se empleó la propuesta de Seyfang y Longhurst (2013) que clasifica a dicha forma de intercambio en: créditos de servicio, esquemas de intercambio mutuo, monedas locales y mercados de trueque.

\section{Aproximación teórica a las monedas complementarias y sociales}

El dinero es una invención social que puede ser identificado en sus diversas formas, no solo por ser utilizado como instrumento sino porque este pone en evidencia un proceso técnico mediante el cual los diversos actores sociales se relacionan y mercantilizan bienes y servicios, es decir el dinero no solo sostiene a las sociedades modernas, sino que involucra un proceso de interacción social (Peixoto, 2003).

Dicho instrumento económico surge desde la edad antigua (Graue, 2009), donde la sociedad utilizaba objetos que contenían validez y eran aceptados para realizar transacciones considerando la calidad, cantidad y propiedades como: durabilidad, valor único y aceptación social (Smith, 1994; Marx, 2017; Graue, 2009; Schettino, 2015). Por ejemplo: en Abisinia el medio de cambio y comercio era la sal, en la India eran una clase de conchas, en Terranova el bacalao seco, en Virginia el tabaco, en las Indias Occidentales el azúcar, 
en Escocia los clavos (Smith, 1994). Sin embargo, en la Inglaterra primitiva, el ganado y los esclavos eran utilizados como dinero, no obstante, su valor quedaba fijado por la ley. En la Edad Media la pimienta era un valor habitual, mientras que en la primera posguerra en Alemania eran utilizados los cigarrillos (Berstein, 2002). Por su parte, en México se manejaba el cacao, jade o jadeíta (chalchihuis), mantas de algodón (patolcuachtli), obsidiana para crear armas y utensilios, hachuelas de cobre en forma de la letra tau griega $(\tau)$, entre otros (Graue, 2009; Banco de México, 2020).

Debido a que dichas mercancías no cumplían con las cinco características: durable (capaz de circular en un estado aceptable por un periodo de tiempo), transportable (facilidad de trasladar el dinero con un valor sustancial), divisible (capacidad de subdividirse en pequeñas partes sin que pierda su valor para adquirir cualquier mercancía), homogéneo (tener un valor similar a las demás unidades de dinero) y de emisión controlada (controlar el dinero para mantener su valor, ofertar la cantidad suficiente de dinero y evitar su falsificación) (Banxico, 2019). Se comenzó a constituir cerca de los 700 a.C. una innovación ingeniosa que soslayaba pureza, denominadas monedas, las cuales eran definidas en oro. En particular en la ciudad francesa Troyes se utilizaba la onza troy, los egipcios utilizaban los lingotes de oro los cuales llevaban la impresión del faraón Menes en el 4000 a.C, sin embargo, Heródoto menciona que los lidios fueron los primeros en utilizar monedas de oro y plata, que al comienzo del siglo VII a.C. utilizaban depósitos, trozos de electro (una aleación de oro y plata). Además, con el primero de los mermnadas, Giges, revolucionó la emisión privada de dinero y después Ardis en los 660 a.C. instituyó un sistema monetario bimetálico más eficaz, es decir, las primeras monedas reconocibles, las cuales tenían forma redonda y tenían grabadas la cabeza de un león y por detrás la ciudad de Sardes (Berstein, 2002), con el tiempo se crearon los billetes oficiales en el Banco de Inglaterra con el respaldo en oro y plata, ver figura 1.

Figura. 1. El dinero a través del tiempo.

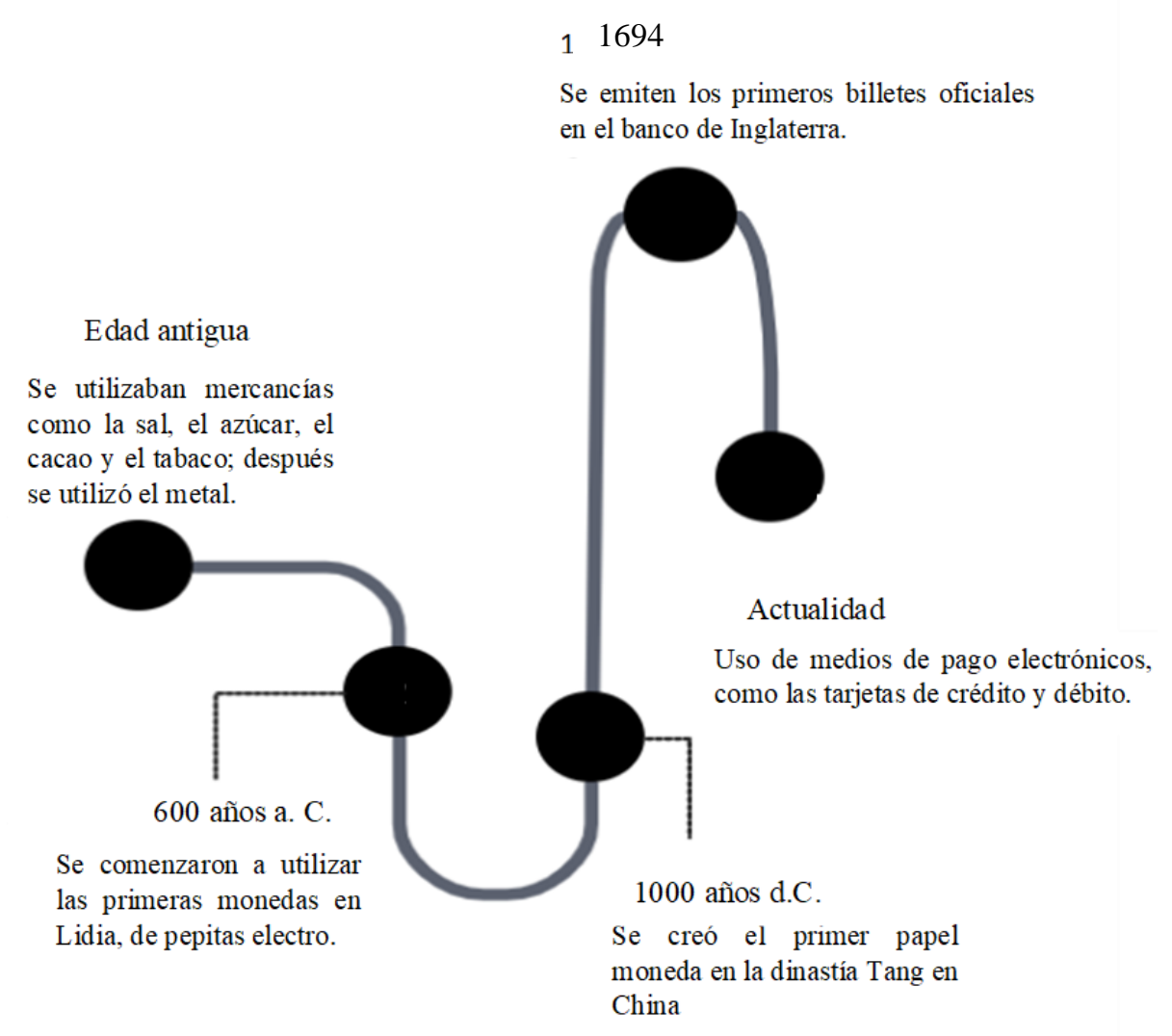

Fuente: Elaboración propia.

Empero, como menciona Berstein (2002) "una sociedad que utiliza el metal como moneda se hallará siempre limitada por la oferta de aquel" (p. 59), por lo tanto, se creó el dinero fiduciario, el cual es decretado por los gobiernos de cada país para que sea legalmente aceptado y funcione como medida de intercambio basado en la confianza de la sociedad hacia el intercambio del papel moneda (McLeay, Radia, Thomas, 2015). 
El patrón monetario y estructural mencionado con anterioridad es operado por el sistema monetario de cada país el cual gobierna la circulación y la emisión de billetes y monedas (Méndez, 2004). Debido a sus funciones anteriores el sistema monetario posee cuatro fallas estructurales: el primero referente a la banca, la cual es responsable de la emisión de dinero y créditos, a pesar de ello también son responsables del aumento o disminución de la masa monetaria; la segunda se refiere a las tasas de interés compuesto a futuro no atractivas para los inversionistas; el tercero es la concentración de la riqueza en la sociedad con mayores ingresos, mientras que el restante y la mayoría de la población está sobre endeudada o en situación de pobreza; el cuarto es la generación de competencia entre la sociedad induciendo a tener menores relaciones humanas; mientras que las fallas restantes son la inflación que deriva en el aumento de precios y pérdida del poder adquisitivo, desequilibrio económico establecido en el ajuste de la oferta y demanda, devaluación, deuda externa, entre otras (Lietaer, 2001; Lietaer, Arnsperger, Goerner y Brunnhuber, 2012; Méndez, 2004).

Se considera que, debido a la escasez y las crisis económicas, surgieron las monedas complementarias y sociales principalmente en América Latina y Europa, las cuales han sido creadas por la sociedad con la finalidad de generar un medio para efectuar transacciones, amortiguar las crisis económicas (Orzi, 2017), compensar la insolvencia de la moneda nacional, subsanar el desempleo y la pobreza. Su propósito principal es desarrollar, fortalecer y estimular a la economía social, en otras palabras, beneficiar a la sociedad sin buscar fines de lucro (Lietaer, 2001; Hirota, 2013).

Las monedas complementarias y sociales se refieren al uso de medios de pago paralelos no sustitutivos a la moneda oficial (Primavera, 2003). No obstante, en stricto sensu, es un instrumento fiduciario con valor en función de la confianza de los usuarios, utilizado por un grupo de personas que ejercen las funciones de valor hacia la unidad que circula libremente en una comunidad y es aceptada como medio pago. Para tal efecto, la moneda alternativa es representada como una práctica social con fecha y ubicación histórica (Peixoto, 2003), verbigracia, las alternativas que surgieron en Grecia debido a las fuertes crisis e imposiciones de los organismos internacionales (Orzi, 2017).

Si la confianza es el factor distintivo para validar la aceptación de las monedas alternativas, es porque es un instrumento que involucra la relación social constituida por la reivindicación y canalización de las capacidades productivas de un grupo y/o comunidad, razonada hacia la pluralidad y producción de variantes económicas (Peixoto, 2003), es decir son instrumentos basados en los valores de ayuda mutua, responsabilidad, democracia, igualdad y solidaridad (Lara y Demmler, 2018).

Los acuerdos comunitarios indicados también se conocen como monedas comunitarias, locales o licitación común (Lietaer, 2004); los cuales apoyan y promueven la colaboración entre la sociedad, desarrollo de empresas o economías que tengan como objeto favorecer, brindar y ayudar a la comunidad (Lietaer, 2001).

La clasificación de las monedas complementarias y sociales se basa en cuatro tipos: los créditos de servicio, esquemas de intercambio mutuo, monedas locales y mercados de trueque (Seyfang y Longhurst, 2013) los cuales tienen diferentes funcionamientos, características y unidades monetarias (Ver Tabla 1).

Tabla. 1. Clasificación de las monedas complementarias y sociales.

\begin{tabular}{|c|c|c|c|}
\hline Clasificación & Funcionamiento & Características & $\begin{array}{c}\text { Unidad } \\
\text { Monetaria }\end{array}$ \\
\hline $\begin{array}{l}\text { Créditos de } \\
\text { servicio }\end{array}$ & $\begin{array}{l}\text { 1. Inscripción de miembros a los } \\
\text { servicios que necesitan u ofrecen. } \\
\text { 2. Organización de la coincidencia de } \\
\text { personas } \\
\text { 3. Generación de intercambios entre } \\
\text { participantes a través de créditos de } \\
\text { tiempo. }\end{array}$ & $\begin{array}{l}\text { El tiempo se puede guardar } \\
\text { para el futuro, donar a otros o } \\
\text { comprar servicios de algún } \\
\text { miembro. }\end{array}$ & Tiempo. \\
\hline $\begin{array}{l}\text { Esquemas de } \\
\text { intercambio } \\
\text { mutuo }\end{array}$ & $\begin{array}{l}\text { 1. Se realiza una transacción o se solicita } \\
\text { un crédito. } \\
\text { 2. Los socios registran sus saldos. } \\
\text { 3. Se registra saldo negativo al } \\
\text { comprador y positivo al vendedor. }\end{array}$ & $\begin{array}{l}\begin{array}{l}\text { Se crean al } \\
\text { transacciones }\end{array} \text { o } \begin{array}{r}\text { efectuar } \\
\text { solicitar }\end{array} \\
\text { créditos. } \\
\text { No hay dinero físico ni } \\
\text { intereses. } \\
\text { Las cuentas tienen suma cero. }\end{array}$ & $\begin{array}{l}\text { Registro de } \\
\text { saldos. }\end{array}$ \\
\hline
\end{tabular}




\begin{tabular}{|c|c|c|c|}
\hline $\begin{array}{l}\text { Monedas } \\
\text { locales }\end{array}$ & $\begin{array}{l}\text { 1. La comunidad o empresas generan } \\
\text { una moneda complementaria localmente } \\
\text { aceptada. } \\
\text { 2. Los usuarios pueden adquirir } \\
\text { productos o servicios en empresas o } \\
\text { áreas participantes con esa moneda } \\
\text { creada. }\end{array}$ & 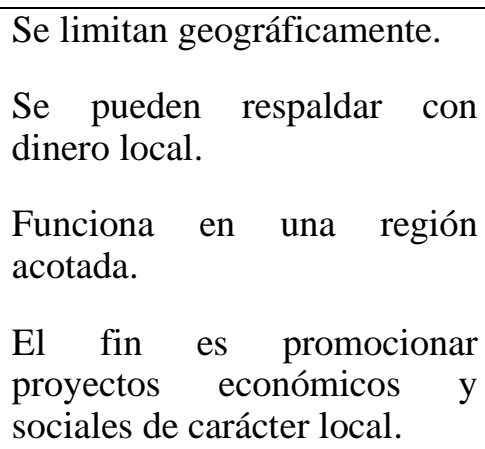 & $\begin{array}{l}\text { Moneda } \\
\text { local. }\end{array}$ \\
\hline $\begin{array}{l}\text { Mercados de } \\
\text { trueque }\end{array}$ & $\begin{array}{l}\text { 1. Las personas realizan un intercambio } \\
\text { directo de producto contra otro producto } \\
\text { o servicio. }\end{array}$ & $\begin{array}{l}\text { Se realizan en eventos } \\
\text { específicos o sitios limitados. } \\
\text { No se usa dinero fiduciario. }\end{array}$ & $\begin{array}{l}\text { Productos o } \\
\text { prestaciones. }\end{array}$ \\
\hline
\end{tabular}

Fuente: Elaboración propia con base en Seyfang y Longhurst (2013), Longhurst (2012), Rodas Carpizo y Rodas Arroyo (2011).

La clasificación de las monedas complementarias que se presenta en la Tabla 1, se constituye en primera instancia por los créditos de servicio, los cuales se refieren a aquellos que tienen una unidad monetaria de tiempo y el funcionamiento de estos se basa en pertenecer a un conjunto de miembros que requieren y ofrecen servicios, para posteriormente efectuar el intercambio entre los participantes a través de créditos estimados en tiempo (Seyfang y Longhurst, 2013; Longhurst, 2012; Rodas Carpizo y Rodas Arroyo, 2011).

Los esquemas de intercambio mutuo funcionan a través del registro de saldos realizado por los socios cuando realizan transacciones o solicitan un crédito, es decir, se registra un saldo negativo cuando la persona efectúa una compra y positivo cuando se concreta una venta, sin la existencia de dinero físico sino saldos registrados de manera virtual donde las cuentas tienen una suma cero (Seyfang y Longhurst, 2013; Longhurst, 2012; Rodas Carpizo y Rodas Arroyo, 2011).

Además, las monedas locales son monedas físicas que pueden ser o no respaldadas por el dinero fiduciario, sin embargo, funcionan en un espacio geográfico determinado, creando tres ventajas principales (Blanc, 2002; Seyfang y Longhurst, 2013; Longhurst, 2012; Rodas Carpizo y Rodas Arroyo, 2011):

- Son proveedoras de un medio de intercambio que se crea en contraste con las necesidades económicas de la comunidad,

- $\quad$ Estas no tienen tasas de interés.

- Estimulan y promueven a la economía local, donde los usuarios tienen la libertad de adquirir productos o servicios en empresas, mercados, comunidades o áreas participantes.

Por su parte, el trueque es una clasificación basada en una unidad monetaria de bienes o servicios, en la cual se realizan intercambios directos de productos o prestaciones en eventos específicos o sitios determinados, con la prohibición de utilizar dinero legal como medio de intercambio (Seyfang y Longhurst, 2013; Longhurst, 2012; Rodas Carpizo y Rodas Arroyo, 2011).

Las monedas complementarias y sociales en todas sus tipologías son herramientas que fomentan la sostenibilidad y desarrollo de la humanidad, ya que proporcionan valores como la cooperación, sustentabilidad y confianza que el dinero convencional no aporta principalmente en crisis económicas. Además, estas alternativas fomentan la economía de manera paralela y crean sociedades más justas y equitativas (Corrons, 2007).

\section{Resultados: las monedas complementarias y sociales en las comunidades mexicanas}

En México desde los años setenta del siglo XX se han ido desarrollando proyectos de economía solidaria, principalmente en entornos rurales y agrícolas. Fue hasta los años noventa cuando se incrementaron las experiencias, proyectos y trabajos relacionados con las monedas complementarias y sociales, pese a ello, estos son considerados escasos (Chaparro, 2014).

En cuanto al contexto mexicano, puede decirse que existe una diversidad de realidades socioeconómicas, debido a que, de los más de 126 millones de habitantes, 52 millones se encuentran en situación de pobreza; es decir, un $34.5 \%$ se encuentra en situación de pobreza moderada, 7.4 está en pobreza extrema, el $29.3 \%$ es vulnerable por carencias sociales y el $6.9 \%$ es población vulnerable por ingresos. Pueden mencionarse otros aspectos que dan cuenta de la situación en que vive la mayoría de la población, tales como: 21.1 millones de 
personas se encuentran en rezago educativo, 20.2 millones de personas viven con carencia de acceso a los servicios de salud, 71.7 millones de personas carecen del acceso a la seguridad social, mientras que 13.8 millones de personas carecen de calidad y espacios de vivienda. Adicionalmente, puede señalarse que 61.1 millones de personas perciben un ingreso insuficiente para poder adquirir las canastas alimentarias y no alimentaria, es decir, el ingreso familiar no solo es precario, sino que es extremadamente concentrado, ya que el $21.9 \%$ equivalente a 27.4 millones de personas pertenecen a la población no pobre y no vulnerable, dicho de otra manera, clase media baja, media y alta (CONEVAL, 2019, 2020).

La pobreza se agudiza en el sureste del país principalmente en las entidades federativas de Chiapas, Campeche, Guerrero, Oaxaca, Veracruz, Tabasco, Puebla, Michoacán, Morelos, Guanajuato, Hidalgo y México; principalmente, entre la población indígena que viven en zonas rurales, los adultos mayores, la población con discapacidad y los niños, niñas y adolescentes principalmente, los que se encuentran en situación de calle, con malnutrición, sin acceso a servicios de educación, salud, seguridad social y vivienda (CONEVAL, 2019, 2020).

Por consiguiente, la introducción de las monedas sociales y complementarias que han surgido entre la sociedad mexicana, no proceden de iniciativas públicas o de aportes científicos, sino de la vida cotidiana, impulsada por y para la comunidad.

Algunas experiencias datan de la década de 1990, cuando se introdujo uno de los principales instrumentos alternativos de pago llamado sistema Boja, que en Nanhú significa dinero. Dicho medio de pago fue utilizado en tiendas de red abastecedoras de productos de primera necesidad en el Valle del Mezquital, Hidalgo; en esa época la región era una de las zonas más marginadas de México (Mazariegos Henkie, 2011). Posteriormente, surgió el Tlaloc y la Mixuca en la ciudad de México, mientras que en Veracruz se incursionó con el Túmin. De manera que en México se cuenta con aproximadamente diecisiete monedas comunitarias (Chaparro, 2014), aunque hoy en día no todas se encuentran vigentes.

Enseguida, se presentan los proyectos o experiencias de monedas complementarias y sociales que se encuentran vigentes, las mismas son clasificadas en cuatro secciones: los créditos de servicio, los esquemas de intercambio mutuo, las monedas locales y finalmente los mercados de trueque.

\subsection{Créditos de servicio}

En esta categoría se sitúan los bancos de tiempo debido a que son iniciativas comunitarias en donde se hace uso del tiempo como unidad de moneda local, en el cual los usuarios dan y reciben servicios o ayuda a través de créditos de tiempo, sin la necesidad de utilizar dinero nacional (Seyfang, 2004). En otros términos, las personas intercambian sus habilidades con un grupo de personas inscritas al sistema y que aceptan a este medio de cambio como una alternativa económica solidaria, como menciona la Asociación de Desarrollo de los Bancos de Tiempo: no se trata de vender tiempo sino establecer relaciones para reformar su comunidad, ya que todos los miembros son importantes (BdTonline, 2018).

En México se encuentran principalmente ocho bancos de tiempo: el Banco de Tiempo CooperAndo, Banco de Tiempo de Guadalajara, Banco de Tiempo Solidario de México, Banco de Tiempo Tapachula y Banco de Tiempo Mérida Yucatán, Banco de Tiempo Mx, Banco de Tiempo Patio Vivo, Banco de Tiempo Transición Tapachula (Ver tabla 2).

Tabla. 2. Bancos de tiempo en México.

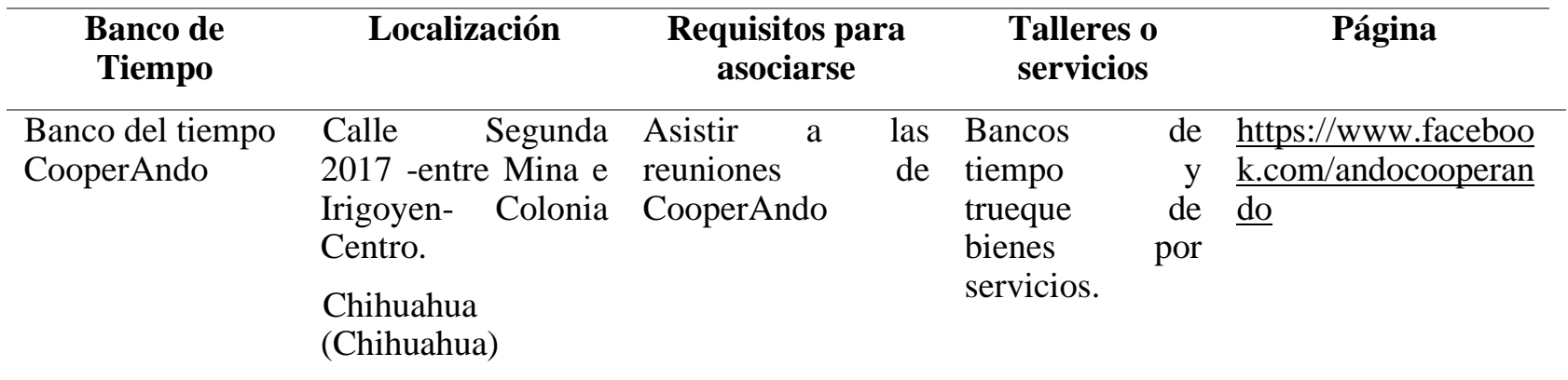




\begin{tabular}{|c|c|c|c|}
\hline $\begin{array}{l}\text { Banco de Tiempo } \\
\text { de Guadalajara }\end{array}$ & Guadalajara & $\begin{array}{l}\text { Contactar al banco } \\
\text { y este busca quien } \\
\text { necesita el servicio. }\end{array}$ & \begin{tabular}{l}
\multicolumn{2}{c}{ Servicios } \\
de personas que \\
puedan y deseen \\
realizar, todos \\
los servicios \\
tienen el mismo \\
valor.
\end{tabular} \\
\hline
\end{tabular}

\begin{tabular}{|c|c|c|c|c|}
\hline $\begin{array}{l}\text { Banco de Tiempo } \\
\text { solidario de } \\
\text { México }\end{array}$ & Ciudad de México & $\begin{array}{lr}\text { Enviar } & \text { mensaje } \\
\text { privado } & \text { con } \\
\text { nombre } & \text { completo, } \\
\text { fecha } & \text { de } \\
\text { nacimiento y correo } \\
\text { electrónico. }\end{array}$ & $\begin{array}{l}\text { Servicios } \\
\text { presenciales } \\
\text { como jardinería, } \\
\text { plomería, } \\
\text { mantenimiento, } \\
\text { ayuda en } \\
\text { mudanzas, } \\
\text { clases } \\
\text { particulares etc. }\end{array}$ & $\frac{\text { https://www.faceboo }}{\text { k.com/BTSM2016/ }}$ \\
\hline $\begin{array}{l}\text { Banco de Tiempo } \\
\text { Tapachula }\end{array}$ & Tapachula, Chiapas & $\begin{array}{l}\text { Realizar cuenta y } \\
\text { ver tutorial de uso }\end{array}$ & $\begin{array}{l}\text { Los que puedan } \\
\text { ofrecer. }\end{array}$ & $\begin{array}{l}\text { https://transiciontapa } \\
\text { chula.wordpress.com } \\
\text { /bancotiempo/ }\end{array}$ \\
\hline
\end{tabular}

\begin{tabular}{|c|c|c|c|c|}
\hline $\begin{array}{l}\text { Banco de Tiempo } \\
\text { Mérida-Yucatán }\end{array}$ & Mérida, Yucatán & $\begin{array}{l}\text { Inscripción, } \\
\text { entrevista y } \\
\text { después dan el alta. }\end{array}$ & $\begin{array}{l}\text { Masa para } \\
\text { Pizza, } \\
\text { construcción de } \\
\text { hornos solares, } \\
\text { limpiar parques, } \\
\text { etc. }\end{array}$ & $\begin{array}{l}\text { http://www.bdtmerid } \\
\text { ayucatan.org/\#home }\end{array}$ \\
\hline $\begin{array}{l}\text { Banco de tiempo } \\
\text { MX }\end{array}$ & $\begin{array}{l}\text { Toluca, Estado de } \\
\text { México }\end{array}$ & 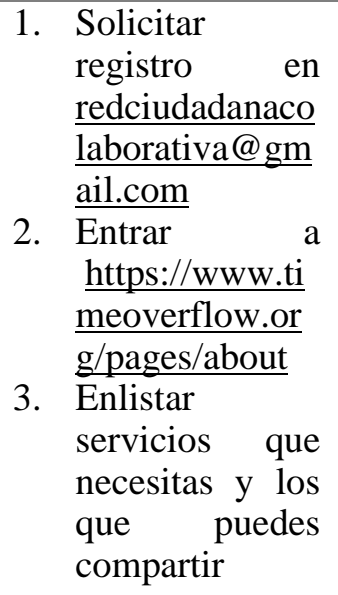 & $\begin{array}{l}\text { Todos los } \\
\text { servicios que } \\
\text { los usuarios } \\
\text { puedan ofrecer }\end{array}$ & $\begin{array}{l}\text { https://asifineart.wixs } \\
\text { ite.com/redcco }\end{array}$ \\
\hline $\begin{array}{l}\text { Banco del } \\
\text { Tiempo Patio } \\
\text { Vivo }\end{array}$ & Ciudad de México & $\begin{array}{l}\text { Registro en } \\
\text { patiovivomx@gmai } \\
\text { l.com y conteo de } \\
\text { saldos en horas }\end{array}$ & $\begin{array}{l}\text { Servicios que } \\
\text { motiven la } \\
\text { creatividad y } \\
\text { participación en } \\
\text { la comunidad }\end{array}$ & $\begin{array}{l}\text { https://www.faceboo } \\
\text { k.com/patiovivomx }\end{array}$ \\
\hline $\begin{array}{l}\text { Banco de tiempo } \\
\text { Tapachula }\end{array}$ & Tapachula, Chiapas & $\begin{array}{l}\text { Registrar } \\
\text { cuenta } \\
\text { https://www.timeov } \\
\text { erflow.org/ }\end{array}$ & $\begin{array}{l}\text { Servicios } \\
\text { educativos } \\
\text { culturales }\end{array}$ & $\begin{array}{l}\text { https://transiciontapa } \\
\text { chula.wordpress.com } \\
\text { /bancotiempo/ }\end{array}$ \\
\hline
\end{tabular}

Fuente: Elaboración propia.

Todos los bancos de tiempo necesitan un plan o modelo de funcionamiento, es decir para que sean eficaces requieren tener un equipo gestor, los cuales llevan la responsabilidad de la creación y gestión del tiempo, registro de transacciones, tareas o actividades del banco y la colaboración con otras asociaciones. Para que los bancos continúen vigentes requieren en primer lugar estar abiertos a nuevos miembros a los cuales se les aplican entrevistas presenciales o virtuales, con la finalidad de conocer las necesidades y 
habilidades del reciente socio, y finalmente escuchar y atender las ideas recientes de los socios, los cuales se reúnen en lugares o instalaciones culturales (Gisbert, 2010).

\subsection{Esquemas o mercados de intercambio mutuo}

El Intercambio de Bienes y Servicios S.A de C.V (IBS) es un mercado de intercambio mutuo nacional de bienes y servicios, originario de Guadalajara, Jalisco en 1995 y en la actualidad se ha extendido hasta Manzanillo y Puerto Vallarta. El funcionamiento de este esquema es a través de las reuniones entre empresas que acceden a realizar transacciones entre sí, las cuales se solventan con los bienes y servicios brindados, mientras que las actividades comerciales son coordinadas, controladas y reguladas a través de créditos atribuidos por la administración para registrar los intercambios realizados en un periodo de tiempo, ver figura 2 (IBS, 2018).

Figura. 2. Funcionamiento del IBS.

\begin{tabular}{|c|c|c|}
\hline $\begin{array}{l}\text { IBS entrega a sus nuevos } \\
\text { afiliados: tarjetas de } \\
\text { identificación, vocucher, } \\
\text { directorio de afiliados y un } \\
\text { contrato del cual se } \\
\text { desprende un crédito. }\end{array}$ & 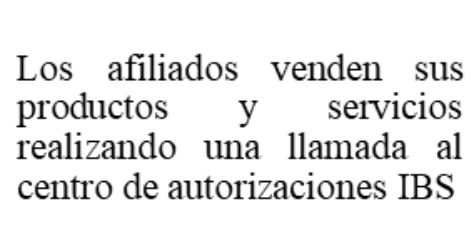 & $\begin{array}{l}\text { Los afiliados que desean } \\
\text { realizar la transacción se } \\
\text { comunican para negociar el } \\
\text { precio, una vez de acuerdo, } \\
\text { indican al cajero que van a } \\
\text { pagar con IBS. }\end{array}$ \\
\hline
\end{tabular}

\begin{tabular}{|l|l|}
\multicolumn{1}{c|}{$\downarrow$} \\
$\begin{array}{l}\text { La cajera llama al centro de } \\
\text { autorización indicando el } \\
\text { comprador, vendedor y monto } \\
\text { de transacción, para que el } \\
\text { sistema verifique si tienen } \\
\text { capacidad de pago con IBS }\end{array}$
\end{tabular}$\rightarrow \begin{aligned} & \text { Teniendo la capacidad,se } \\
& \text { plasma en el vocucher para } \\
& \text { que el afiliado firme y } \\
& \text { obtenga la mercancía. }\end{aligned}$

Fuente: Basada en Intercambio de Bienes y Servicios IBS (2018).

Este tipo de intercambios es distinto al trueque, en primer lugar, porque se utiliza una moneda virtual entre los socios, en la que se establece cual es el crédito o capacidad de cada empresa de acuerdo con el IBS, en segundo lugar, las operaciones son multidireccionales, es decir, se pueden realizar intercambios con distintos usuarios, ya que al final compran lo que necesitan y pagan con lo que ellos producen y finalmente hay un único intermediario central. Conjuntamente, los afiliados tienen tres ventajas: la primera es que incrementan las ventas al usar IBS y utilizan mejor su capacidad instalada, la segunda es la mejoría de la liquidez porque paga con IBS, exentos de impuestos al no utilizarse dinero legal de por medio y la tercera es que existe abatimiento de costos porque pagan con insumos y dejan el efectivo para solventar otros gastos externos (IBS, 2018).

\subsection{Monedas locales}

Las monedas locales surgen incentivadas por la sociedad en un área o región determinada como: universidades, organizaciones civiles, mercados o tianguis, zonas indígenas o de productores pequeños, entre otros; las cuales se crean para estimular la economía, apoyar a grupos vulnerables, integrar a partes de la sociedad afectada, para paliar la crisis económica o de seguridad en una zona o bien como una actividad académica en las universidades. En México se identifican monedas, tales como: el Tlaloc, Túmin, Fausto, Itacate, Kuni, Mixiuhca, Almanatli y Cajeme.

\subsubsection{Tlaloc}

El Tlaloc es una moneda comunitaria creada en la zona metropolitana de México, por la organización civil: Promoción del Desarrollo Popular (PDP), encabezada por Luis Lópezllera y que ha sobrevivido hasta el día de hoy (Santana Echeagaray, s.f; Cortés, 2008). 
En sus inicios esta moneda facilitó el intercambio entre 50 productores y consumidores, que se encontraban en la crisis económica de México de 1995. Los miembros fundadores comenzaron a realizar intercambios mutuos y para facilitarlos crearon el Tlaloc, una moneda autónoma de la moneda nacional, la cual equivalía a una hora de trabajo, que por el tipo de cambio se fue transformando con los años, aunque el mínimo se estableció alrededor de tres dólares (Santana Echeagaray, s.f; Más de MX (2017).

El funcionamiento de estas monedas inicia cuando los participantes o usuarios firman una carta compromiso junto con la cual reciben 10 tlalocs para realizar intercambios, cuando se realizan las transacciones, se elabora un balance entre las compras y ventas realizadas para llevar un registro; en caso de retirarse los socios tienen que devolver la cantidad de crédito que obtuvieron (Santana Echeagaray, s.f).

Esta moneda tuvo un auge sin precedentes, la cual propició en un principio a realizar intercambios semanales y fomentar el apoyo mutuo entre los productores, sin embargo, dejaron de asistir gradualmente por lo que implementaron la presencia en ferias anuales, expo ventas y mercados solidarios, continuando vigentes hasta la fecha actual y dando apertura a la gente que desee anexarse al sistema Tlaloc sin necesidad de vender o comprar productos. Gracias a estas reuniones, la red Tlaloc ayudó en la creación de otras monedas comunitarias (Santana Echeagaray, s.f y Cortés, 2008).

\subsubsection{Túmin}

La palabra Túmin como menciona Castro Soto (2016) y Hirota (2017) significa dinero en totonaca, pero está relacionada con la palabra "tomín" en castellano, es la moneda social más próspera y fuerte de México, y la cual se puso en circulación en el 2010.

La Junta del Buen Gobierno (2014) menciona que el Túmin no es una moneda que nació de un proyecto institucional, pero sí apoyado por estudiantes y egresados de Universidad Veracruzana Intercontinental, lo que daba un respaldo moral y credibilidad para el pueblo, ya que esta moneda alternativa es utilizada para pequeños productores de bienes y servicios, con la finalidad de incluir a las comunidades indígenas (Nájar, 2012).

Los túmines son respaldados por las personas que hacen uso de ellos, y es un sistema de trueque donde el dinero no es lo que vale sino los productos y/o servicios que las personas dan a cambio a través del uso de túmines (Nájar, 2012; Ochoa Martinez, 2016), teniendo presencia no solo en Veracruz sino en otros estados como Chiapas, Puebla, Hidalgo y Ciudad de México (Robles, 2013).

Esta moneda es importante porque tuvo una denuncia realizada por el Banco de México (Telesur, 2012; Nájar, 2012) ya que sintieron una amenaza real al sistema monetario nacional, sin embargo, el Tumín como menciona Juan Castro Soto en el video realizado por Telesur (2012) no remplaza al peso como acusaba el Banco de México, puesto que solo se emplea en una comunidad, región o localidad específica, no cobra intereses, no es oficial, público, privado ni obligatorio, simplemente cooperativo.

\subsubsection{Fausto}

Fausto es una moneda creada en la Universidad Nacional Autónoma de México y se utiliza para la feria semestral de Multritueque Fausto en la facultad de economía de la UNAM, dicha moneda tiene como objetivo que los alumnos universitarios comprendan el funcionamiento del dinero y las alternativas para adquirir productos como libros, folders, nieves, arreglos florales y pláticas (Correa, s.f).

El funcionamiento del Fausto comienza cada vez que se reparten 30 faustos por prosumidor (La coperacha, 2016), el cual según Marshall y Barringtones es cuando un consumidor puede tomar ambos roles él de consumidor y productor a la vez (Islas, 2008), en total circulan 1500 Faustos intercambiables en la feria semestral entre prosumidores; los que no pertenecen a esta categoría pero desean participar se les hace entrega de un Quetzal equivalente a un peso mexicano para que puedan participar en el proyecto (La coperacha, 2016).

\subsubsection{Itacate}

El Itacate inició en el centro de la ciudad de Guadalajara en el 2013 por productores de bienes naturales, artesanos e indígenas (Correa, 2013). En un principio esta alternativa fue impulsada por la Red Tláloc, conformada por la Unidad de Apoyo a Comunidades Indígenas de la Universidad de Guadalajara, pero es principalmente utilizada por productores Jaliscienses en el Corredor Cultural Expiatorio, los cuales se reúnen cada fin de semana (Robles, 2013).

El billete "itacate" está aprobado por la Red Mexicana de Comercio Comunitario y se le da el valor que la comunidad apruebe, funciona cobrando el $20 \%$ de itacates por valor real del producto, por ejemplo: un producto cuesta 30 pesos entonces equivale a 9 itacates (Robles, 2013). 


\subsubsection{Kuni}

El Kuni es una moneda empleada en Querétaro en la zona Nhanhú principalmente para los productores de maíz orgánico, hortalizas, jabones, artesanías, pan artesanal, entre otros servicios. Kuni significa movimiento o energía en nhanhú (Correa, 2013).

En otras palabras, es un vale que sirve para generar confianza al realizar trueque de bienes y servicios específicamente solidarios y ecológicos. Al cual se le da la unidad de valor representativa o en proporción a los bienes y trabajos que ofrecen las personas pertenecientes a esa red, donde el valor de los productos dependerá de la autonomía de los socios, ya que entre más autónomos sean respecto al valor, mayor número monedas Kunis reciben (Vale de Multitrueque Kuni, 2015).

Los intercambios que se realizan a través de este vale de multitrueque deben ser voluntarios y ocurren en un mercado abierto, es decir los kuniteros pueden intercambiar en cualquier momento y lugar donde tiene validez la moneda siempre y cuando se llegue a acuerdos de calidad, precio y tiempo. La entrega de los Kuni se realiza directamente entre el beneficiario (demandante) y el generador (oferente) antes o después de la entrega del producto o realización del servicio (Vale de Multitrueque Kuni, 2015). Los productos y servicios que se ofrece la comunidad del Kuni, son los siguientes: ver Tabla 3.

Tabla. 3. Productos y Servicios que se pueden adquirir con el Kuni.

\begin{tabular}{|c|c|c|}
\hline \multirow[t]{5}{*}{ Productos } & $\begin{array}{l}\text { Alimentos } \\
\text { bebidas }\end{array}$ & $\begin{array}{l}\text { Frutas, verduras, bebidas (café yachil, té chau, galoria, manzanilla), comida } \\
\text { preparada (sal vegetal, mermelada, nuevo producto, galletas artesanales y pan } \\
\text { de elote }\end{array}$ \\
\hline & Salud y belleza & $\begin{array}{l}\text { Cremas, shampoo, gel de ducha, aceite de almendras, jabones, pasta de } \\
\text { dientes sin flúor, chaya, etc. }\end{array}$ \\
\hline & Para la tierra & $\begin{array}{l}\text { Macetas de unicel Reciclado, semillas para hortalizas, plantas, lombriz roja } \\
\text { californiana }\end{array}$ \\
\hline & Artesanías & $\begin{array}{l}\text { Diseño de artesanías a través de fibras y maderas naturales, reciclado de } \\
\text { unicel, taller de laudería }\end{array}$ \\
\hline & Materia Prima & Semillas para hortalizas \\
\hline \multirow[t]{4}{*}{ Servicios } & Medicina & Limpieza dental, psicoterapia, sesión de biomagnetismo \\
\hline & $\begin{array}{l}\text { Clases, cursos y } \\
\text { asesorías }\end{array}$ & $\begin{array}{l}\text { Regularizaciones escolares, taller de huertos en casa, taller y asesorías de } \\
\text { economía solidaria y buen vivir, }\end{array}$ \\
\hline & Oficios & Ecoturismo, carpintería, turismo \\
\hline & Rentas & Camionetas y eco alojamiento \\
\hline
\end{tabular}

Fuente: Basado en Vale de Multitrueque Kuni (2015).

Es una red de Multitrueque efectuada en la Ciudad de México y donde los usuarios utilizan como moneda "Mixiuhca" basada en la reciprocidad y crédito comunitario para realizar transacciones (La Escuelita, 2014).

Este tipo de dinero complementario tiene como base la confianza de los miembros para aceptarla como medio de pago de productos y servicios, por ende, el Mixiuhca es una clase de dinero creado por una comunidad; la página de Feria Multitrueke menciona que la unidad de cuenta de este sistema monetario alternativo es la alegría (Ferial Multitrueke, 2018).

El funcionamiento de la Mixhuca comienza en cuanto las personas depositan su confianza, aceptación en este instrumento para dar surgimiento a su valor, una vez que hay confianza las personas se disponen a adquirir bienes y/o servicios, cuando satisfacen una necesidad firman el papel denominado Mixhuca para registrar la transacción realizada (Ferial Multitrueke, 2018).

\subsubsection{Amanatli}

En el tianguis de multitrueque de San Miguel de Allende, Guanajuato nació la moneda comunitaria llamada Amanatli, tal moneda fue impulsada por Ariel Garibaldi quien participó en los tianguis de multitrueque de Mixihuca (La Coperacha, 2015).

Garibaldi menciona que la circulación de dicha moneda es importante y benéfica para la comunidad, ya que intercambian artesanías, pan, dulces, galletas, medicina alternativa, artículos de salud, belleza, entre otras cosas. Al igual que en las monedas sociales mencionadas con anterioridad es de vital importancia que en cada uno de los intercambios efectuados exista la confianza entre el consumidor y productor, además de afecto y buena voluntad (La coperacha, 2015). 


\subsubsection{Cajeme}

El Cajeme surge de una iniciativa realizada por la Cruz Roja de Sonora, esta moneda en un principio comenzó como una red comunitaria conformada por diversas instituciones sociales y empresarios. La cual funcionó como un vale de trueque que complementaba el sueldo del personal (personal de organizaciones civiles, jardineros, plomeros, etc.), donde estos podían intercambiarlos por bienes en un bazar, lamentablemente el bazar cerró (Santana Echeagaray, s.f). No obstante, esta se convirtió en una moneda "cajeme" que se intercambiaba por pesos; utilizada por empresarios para apoyar causas sociales, además eran aceptados en locales comerciales y gasolineras. Sin embargo, esta moneda perdió el sentido social y ahora se conoce como Tomi que significa dinero (lengua yaqui), el tomi sirve para que los empresarios puedan intercambiar sus vales por artículos en bazares. Empero deben tener cuidado de no solo fortalecer al comercio local sino también a los pequeños emprendedores para que puedan crecer conjuntamente generando recursos e intercambiar bienes y servicios (Santana Echeagaray, s.f.).

\subsection{Mercados de trueque}

El trueque para Rodas Carpizo y Rodas Arroyo (2011) "es el cambio directo de un producto o prestación contra otro producto o prestación "(p.100). El fin de estos es intercambiar bienes y servicios en un evento específico o sitio limitado, anulando el uso de la moneda legal, este tipo de trueques funcionan cuando los socios se reúnen y consumen en ese mercado o club (Seyfang y Longhurts, 2013). El trueque cuenta con las siguientes dificultades para efectuarse correctamente y perdurar, ver tabla 4.

Tabla. 4. Inconvenientes del trueque.

\begin{tabular}{|l|l|}
\hline \multicolumn{1}{|c|}{ Inconveniente } & \multicolumn{1}{|c|}{ Descripción } \\
\hline $\begin{array}{l}\text { Dificultad de coincidencia en los deseos } \\
\text { respectivos de quienes realizan el trueque }\end{array}$ & $\begin{array}{l}\text { No están de acuerdo con los productos de } \\
\text { intercambio }\end{array}$ \\
\hline $\begin{array}{l}\text { Dificultad de concordancia en el valor de los } \\
\text { bienes sujetos al intercambio }\end{array}$ & Creen que el valor de sus productos es mayor \\
\hline Dificultad de transportación & $\begin{array}{l}\text { Dificultad al cargar y transportar grandes } \\
\text { volúmenes de mercancías }\end{array}$ \\
\hline $\begin{array}{l}\text { Dificultad de establecer relaciones de intercambio } \\
\text { en diversidad de mercancías }\end{array}$ & $\begin{array}{l}\text { El trueque es un valor entre mercancías de dos en } \\
\text { dos, carece de poder en forma global del valor de } \\
\text { un bien específico }\end{array}$ \\
\hline Dificultad de divisibilidad & Es difícil dividir los productos \\
\hline Dificultad de durabilidad & $\begin{array}{l}\text { Descomposición de algunos productos, se debe } \\
\text { efectuar en tiempo específico }\end{array}$ \\
\hline
\end{tabular}

Fuente: Basada en Rodas Carpizo y Rodas Arroyo (2011).

Los mercados de trueque son espacios en donde se realizan intercambios de bienes en su mayoría directos y pocas veces indirectos. En México algunas de las monedas complementaria, y sociales, especificando las comunitarias se han creado a través de una red de trueque (López y Benito, 2017), algunos son: la Red de Multitrueque Tlaloc, la Red Multitrueque Mixiuhca, la Red de Transición Querétaro, el Multitrueke Amanatli y el Tianguis de Economía Solidaria de Teotihuacán (Tabla 5). 
Tabla. 5. Mercados de trueque en México.

\begin{tabular}{|c|c|c|c|c|}
\hline Mercado de trueque & $\begin{array}{l}\text { Fecha de } \\
\text { creación }\end{array}$ & Localización & Periodicidad & $\begin{array}{l}\text { Moneda } \\
\text { instituida }\end{array}$ \\
\hline $\begin{array}{l}\text { Red de Multitrueque } \\
\text { Tlaloc }\end{array}$ & 1995 & Ciudad de México & Sistémica & Tlaloc \\
\hline $\begin{array}{l}\text { Red Multitrueque } \\
\text { Mixiuhca }\end{array}$ & 2010 & Ciudad de México & $\begin{array}{l}\text { Feria mensual y } \\
\text { sistémica }\end{array}$ & Mixhuca \\
\hline $\begin{array}{l}\text { Red de transición } \\
\text { Querétaro }\end{array}$ & 2012 & Querétaro & Sistémica & Kuni \\
\hline Multitrueke Amanatli & 2014 & $\begin{array}{l}\text { San Miguel de } \\
\text { Allende, Guanajuato }\end{array}$ & Feria mensual & Amanatli \\
\hline $\begin{array}{l}\text { Tianguis de Economía } \\
\text { Solidaria de } \\
\text { Teotihuacán }\end{array}$ & 2017 & $\begin{array}{l}\text { Teotihuacán, Estado } \\
\text { de México }\end{array}$ & Ferial & Iztli \\
\hline
\end{tabular}

Fuente: Elaboración propia.

Uno de los mercados de trueque directos (los que se basan únicamente en el intercambio inmediato de bienes) es por ejemplo, el mercado de trueque de la Secretaría del Medio Ambiente del Gobierno de la Ciudad de México (SEDEMA, 2016), el que consiste en intercambiar residuos inorgánicos (1 kg a $10 \mathrm{~kg}$ como máximo), como pueden ser latas de aluminio, latas de fierro, envases Tetra Pak, botellas de PET, papel y cartón y botellas de vidrio por productos de origen agrícola local, este trueque se realiza el segundo domingo de cada mes, sin embargo, en este hacen entrega de puntos verdes no acumulables para cambiarlos ese día, existen otros tipos de mercados de trueques como: el trueque de bicicletas en la Ciudad de México, trueque en el carnaval de Papalotla de Xicohténcatl en Tlaxcala, el Trueque de Cholula, Puebla ( Galicia, 2016), además en ese mismo estado se encuentra el trueque realizado en el tianguis del municipio de Tepeaca en el cual no utilizan monedas de por medio (Avelino Morales, 2014), así como el tianguis de trueque y arte alternativo de la ciudad de México, entre otros.

De manera que, al recolectar la información, se identificaron veintitrés proyectos alternativos de monedas complementarias y sociales en México, sintetizado en la tabla 6, por su tipo de clasificación, proyectos y su tipo de respaldo.

Tabla. 6. Resultados de monedas complementarias y sociales por clasificación.

\begin{tabular}{|l|l|l|}
\hline $\begin{array}{c}\text { Clasificación Monedas } \\
\text { complementarias }\end{array}$ & \multicolumn{1}{|c|}{ Proyectos } & Tipo de respaldo \\
\hline Créditos de servicio & $\begin{array}{l}\text { Banco del tiempo CooperAndo, Banco de tiempo de } \\
\text { Guadalajara, Banco de tiempo solidario de México, } \\
\text { Banco de tiempo Tapachula, Banco de tiempo } \\
\text { Mérida-Yucatán, Banco de tiempo Mx, Banco de } \\
\text { tiempo Patio Vivo y Banco de tiempo Tapachula. }\end{array}$ & $\begin{array}{l}\text { Bienes } \\
\text { servicios. }\end{array}$ \\
\hline $\begin{array}{l}\text { Esquemas } \\
\text { intercambio mutuo }\end{array}$ & der. & $\begin{array}{l}\text { Transacción- } \\
\text { crédito mutuo }\end{array}$ \\
\hline Monedas locales & $\begin{array}{l}\text { Tlaloc, Túmin, Fausto, Itacate, Kuni, Mixiuhca, } \\
\text { Almanatli, Cajeme. }\end{array}$ & Ninguno. \\
\hline Mercados de trueque & $\begin{array}{l}\text { Red multitrueque Tlaloc, Multitrueke Almanatli, } \\
\text { Red multitrueque de Mixiuhca, Red de Transición } \\
\text { Querétaro, Tianguis de Economía Solidaria de } \\
\text { Teotihuacán, Trueque de la SEDEMA, Trueque del } \\
\text { carnaval de Papalotla. }\end{array}$ & $\begin{array}{l}\text { Bienes } \\
\text { servicios }\end{array}$ \\
\hline
\end{tabular}

Fuente: Elaboración propia. 
Las monedas complementarias y sociales benefician a las comunidades, principalmente a personas con vulnerabilidad económica, comunidades indígenas y población en situación de pobreza, proporcionándoles oportunidades y alternativas para satisfacer o complementar algunas de sus necesidades principalmente básicas a través del fomento de la solidaridad de las personas, promoviendo el intercambio, inclusión socioeconómica, estimulando la reactivación de actividades económico sociales y contribuyendo al ingreso de bienes y/o servicios que sirven como sustento suplementario tanto al productor como al consumidor.

\section{Discusión}

Se llevó a cabo un estudio documental para describir e identificar el funcionamiento, uso, características, ventajas y contribuciones de las monedas complementarias y sociales, reconocidas y utilizadas en la República Mexicana. Dado que representan un medio de pago alternativo utilizado por la sociedad, las que emergen en lugares delimitados para contrarrestar los efectos de las crisis económicas, la desigualdad y la pobreza recurrente en México y gran parte del mundo, esto provoca que cada vez las personas incentiven y aumenten el uso de estos instrumentos de intercambio.

Con la investigación se encontró que en México las monedas complementarias y sociales se manifiestan en su mayoría en bancos de tiempo, secundando los mercados de trueque, los cuales generalmente han creado monedas locales para poder llevar a cabo la administración de sus transacciones con mayor fluidez, ser prácticas y en la manera de lo posible, permanentes, siempre y cuando sigan demostrando su utilidad y fiabilidad entre las comunidades donde se desarrollan, finalmente pero no por ello menos importante el esquema de intercambio mutuo IBS. Principalmente han surgido en algunas regiones focalizadas con niveles altos de pobreza y pobreza extrema que se encuentran en los siguientes estados: Veracruz, Chiapas, Puebla, Guanajuato, Hidalgo, Ciudad de México, Estado de México y otras partes de la república con niveles menores, pero no por ello inexistentes de pobreza como Chihuahua, Guadalajara, Querétaro y Sonora.

Las monedas complementarias y sociales en México siguen el esquema tradicional de los grupos precolombinos, esto es con el fin de incentivar la economía regional, local y social de los grupos marginados, generando una dinámica en la economía de estas personas, con base en la confianza y el apoyo mutuo.

Desde los años noventa hubo un acrecentamiento en el registro de las monedas complementarias y sociales como instrumentos de la economía solidaria, con una gran penetración y aceptación en la sociedad mexicana. Correlacionando de manera personal la situación pandémica actual (COVID-19) y la crisis económica, se prevé que los mexicanos lleguen a similar la potencialidad del uso de las monedas complementarias y sociales para facilitar la vida cotidiana, principalmente por la realidad de la restricción de horarios de venta, la disminución de horas de trabajo y salarios, el desempleo y el poco acceso a bienes y/o servicios, incluyendo los indispensables como: salud, vivienda, alimentación, educación, entre otros. Y es que no se equivocan Lietaer (2001) y Orzi (2017) cuando la moneda oficial resulta escasa, hay poco acceso a la economía y se generan crisis, derivando esto en el surgimiento de las monedas complementarias y sociales para darle poder a la sociedad y así amortiguar las crisis económicas y la pobreza como en Argentina, España, Grecia, Venezuela, Cuba, etc.

En la actualidad las monedas complementarias y sociales son de gran relevancia para la sociedad, ya que estas herramientas permiten como menciona Petri, Rana y Cosmin (2010) la regeneración económica, no solo por la habilidad de estas para agregar valor a las comunidades que las utilizan, sino porque facilitan la estabilidad de comercio entre las personas que pertenecen a las redes, sociedades o comunidades; es decir, entre miembros que aceptaron estos medios de pago como acuerdos de confianza en un espacio delimitado con la finalidad de apoyar, estimular la circulación e impulsar las transacciones basándose en la reciprocidad y confianza en su localidad (Lietaer, 2004; Corrons, 2017).

Es decir, promueven la economía local favoreciendo a la creación de empleo, la satisfacción de necesidades socioeconómicas, la redistribución de la renta per cápita de subsistencia para evitar la limitación del poder adquisitivo y la inclusión social, dichos instrumentos contribuyen a reducir la desigualdad y permiten el intercambio económico (Caravaca y González, 2019; Lietaer, 2004).

Es de suma importancia mencionar que no existe legislación clara nacional de las monedas complementarias y sociales, debido a que estas tienen una soberanía y aceptación social, basada en la confianza mutua y uso dentro de las comunidades de manera limitada, es decir tienen sus propias reglas internas; de manera que pueden crear diversos medios de intercambio siempre y cuando los usuarios o comunidades donde tienen aplicación estén de acuerdo.

\section{Conclusión}

El ser humano a través de la historia ha creado instrumentos que encaminan al mejoramiento de su economía. Los resultados muestran que las monedas complementarias introducidas por estos individuos o 
grupos permiten abordar los fallos dentro del paradigma del dinero convencional, es decir crea oportunidades en las crisis y trabaja en conjunto con la economía social.

Las monedas complementarias y sociales no son una nueva invención, sin embargo, han generado un cambio económico, aumentando las oportunidades de innovación en la economía social y en la inclusión de grupos vulnerables como: personas en situación de pobreza, pequeños agricultores y productores, ancianos y niños. En México el número de monedas registradas no son cuantiosas, pero su valor está enfocado hacia la asistencia social.

Las monedas complementarias y sociales introducidas a través del tiempo en México y en el resto del mundo, han tenido generalmente un impacto positivo al incentivar y promover el intercambio, fomentar la solidaridad entre personas, grupos o comunidades, estimular actividades económicas con fines sociales y contribuir al ingreso de bienes y/o servicios que satisfagan algunas de las necesidades de la comunidad. Inclusive cuando aumenta el número de monedas complementarias, la economía social se enriquece en primer lugar por la diversidad de instrumentos que permiten la inclusión de personas vulnerables económicamente y de manera secundaria para acentuar a la banca nacional que estas alternativas son numerosas, no monopolizables, por consiguiente, no pretenden sustituir a la moneda nacional.

Con el estudio se buscó evidenciar las monedas complementarias y sociales suscitadas en México, principalmente el enfoque fue a los créditos de servicio o bancos de tiempo, esquemas de intercambio mutuo, monedas locales y trueque. Estos instrumentos proveen las herramientas para generar cambios en la economía local, sin embargo, deberían de divulgar más sus usos y beneficios para que puedan obtener mayor interés y apoyo social para acrecentar su potencialidad y complementar a la moneda convencional.

Es importante indicar que actualmente los programas alternativos son una fuente de mejoras a la vida social, principalmente en los países con regiones con pobreza extrema o con ingresos desiguales, que conlleva a una economía sustentable, incidiendo directamente en un progreso en los aspectos significativos como lo económico, seguridad social, político, salud, alimentación, entre otros; estableciendo lazos de ayuda mutua.

Esta investigación apuesta al desarrollo de las monedas complementarias y sociales en México para dar alternativas a las disfunciones del sistema económico dominante, ya que se ha demostrado que proporcionan beneficios a regiones en situación de pobreza extrema y marginación.

\section{Referencias bibliográficas}

Avelino Morales, T. (2014) El tianguis de trueque que se niega a morir. El financiero. http://www.elfinanciero.com.mx/sociedad/el-tianguis-de-trueque-que-se-niega-a-morir.

Banco de México (BANXICO) (2019) ¿Qué es el dinero? Sitio del Banco de México: http://educa.banxico.org.mx/economia/dinero-economia.html (Última consulta, 19 de diciembre de 2020).

Banco de México (BANXICO) (2020) Historia de la Moneda y el Billete en México: https://www.banxico.org.mx/billetes-y-monedas/d/\%7B1EEDFA6C-8EDB-B7AD-11B2-528C7B69CC76\%7D.pdf (Última consulta, 20 de enero 2021).

Blanc, J. (2002) Formes et rationalités du localisme monétaire. L'Actualité économique, 78, №3, pp. 347-369. https://doi.org/10.7202/007256ar.

Berstein, P. L. (2002) El oro una historia de obsesión. Barcelona, España: Javier Vergara Editor.

BdTonline (2018) ¿Qué son los bancos de tiempo?. http://www.bdtonline.org/ (Última consulta 18 de diciembre de 2020).

Castro Soto, J. (2016) 10 razones del Túmin. TEDxBarriodelEncino Recuperado de https://www.youtube.com/watch?v=TCKKa_8vG9k.

Chaparro, E. (2014) Monedas comunitarias en contextos solidarios. Una aproximación al sentido del uso del Itacate. Tesis Maestría, ITESO.

Consejo Nacional de Evaluación de la Política de Desarrollo Social (CONEVAL) (2019) Diez años de medición de pobreza multidimensional en México: avances y desafíos en política social. Medición de la pobreza serie 20082018: https://www.coneval.org.mx/Medicion/MP/Documents/Pobreza_18/Pobreza_2018_CONEVAL.pdf (Última consulta, 30 de e nero de 2021).

Consejo Nacional de Evaluación de la Política de Desarrollo Social (CONEVAL). (2020) Informe de la pobreza multidimensional 2008-2018. Una década de medición multidimensional de la pobreza en México: Recuperado el https://www.coneval.org.mx/InformesPublicaciones/InformesPublicaciones/Documents/Informe_Pobreza_20082018.pdf (Última consulta, 30 de enero de 2021).

Correa, P. (2013) La Moneda Autónoma Universitaria, en la UNAM. Claudia Valadez explica el "Fausto" en la Facultad de Economía. Recuperado de https://www.kavilando.org/index.php/2013-10-13-19-52-10/formacionpopular/2194-la-moneda-autonoma-universitaria-en-la-unam-claudia-valadez-explica-el-fausto-en-la-facultad-deeconomia.

Corrons, A. (2017) Monedas complementarias: dinero con valores. Revista Internacional de Organizaciones, $\mathrm{N}^{\circ} 18, \mathrm{pp}$. 109-134. https://doi.org/10.17345/rio18.109-134. 
Cortés, F. (2008) Las monedas sociales. CAJAMAR, N4, pp. 1-52. Recuperado de: https://base.socioeco.org/docs/a4 finanzas_eticas.pdf.

Daly, H. (2008) Growth and development: critique of a credo. Wiley Online Library, 34, N³, pp. 511-518. https://doi.org/10.1111/j.1728-4457.2008.00234.x.

Ferial Mutitreuke (2018) Dinero Comunitario: https://vida-digna.org.mx/multitrueke/comunidad-multitrueke/dinerocomunitario/ (Última consulta, 28 de enero de 2021).

Galicia, T. (2016) Tianguis y trueque. Recuperado de http://www.e-consulta.com/opinion/2016-09-09/tianguis-ytrueque.

Gisbert, J. (2010) Guía Bancos de Tiempo, anexo al libro Vivir sin Empleo. Fuente: Vivirsinempleo.org. Recuperado desde http://base.socioeco.org/docs/guiacreacion_banco_de_tiempo.pdf.

Graue, A (2009) Fundamentos de la Economía. México: Pearson Educación.

Hirota, Y. (2013) Monedas complementarias como herramienta para fortalecer la economía social. Universidad de Valencia. Valencia, España.

Hirota, Y. (2016) Monedas sociales y complementarias (MSC) Ventajas y desventajas de los diferentes modelos. Revista de los Estudios de Economía y Empresa, Oikonomics, N. ${ }^{\circ}$ 6, pp. 1-42. ISSN 2339-9546. Universitat Oberta de Catalunya. http://oikonomics.uoc.edu.

Hirota, Y. (2017) Monedas sociales y complementarias (MSCs) - Sus valores socioeconómicos para distintos stakeholders. Tesis Doctoral, Universidad de Valencia.

Intercambio de Bienes y Servicios S.A. de C.V. (IBS, 2018) Intercambio de bienes y servicios: http://www.ibs.com.mx/ (Última consulta, 28 de enero de 2021).

Islas, J. (2008) El prosumidor. El acto comunicativo de la sociedad de la ubicuidad. Red de Revistas Científicas de América Latina y el Caribe, España y Portugal,11, №1, pp. 29-39. ISSN 0122-8285. Recuperado de https://www.redalyc.org/pdf/649/64911103.pdf.

Junta De Buen Gobierno (2014) Aceptamos Túmin Mercado Alternativo, Economía solidaria y Autogestión. Xalapa, México: Códice: http://base.socioeco.org/docs/_uvi_files_2014_12_libro-aceptamos-tumin-version-final.pdf (Última consulta, 20 de enero de 2021).

Lara, G. y Demmler, M. (2018) Social Currencies and Cryptocurrencies: Characteristics, Risks and Comparative Analysis. CIRIEC-España. Revista de Economía Pública, Social y Cooperativa, No 93, pp. 265-291, DOI: 10.7203/CIRIEC-E.93.10978.

La Coperacha (2015) Nace en San Miguel de Allende la moneda comunitaria, Amanatli: https://www.lacoperacha.org.mx/Nace-moneda-comunitaria-San-Miguel-Allende-Guanajuato-Amanatli.php (Última consulta, 23 de enero de 2021).

La Coperacha (2016) El Fausto en Economía de la UNAM (video): https://www.youtube.com/watch?v=-DqK5p4Wr94 (Última consulta, 24 de enero 2021).

La Escuelita (2014) Dinero comunitario: http://vida-digna.org.mx/escuelita/dinero-comunitario/ (Última consulta 30 de enero 2021).

Lietaer, B. (2001) The Future of Money-Creating New Wealth,Workanda Wiser World. Londres: Random House.

Lietaer, B. (2004) Complementary Currencies in Japan Today: History, Originality and Relevance. International Journal of Community Currency. Research. Vol.8, pp.1-23.

Lietaer, B., Arnsperger, C., Goerner, S., Brunnhuver, S., (2012) Money and Sustainability-The Missing Link. Devon, Reino Unido: Triarchy Lress.

Longhurst, N. (2012) The Totnes Pound: A grassroots technological niche in Davies, A. Enterprising Communities: Grassroots sustainability innovations. (Bingley: Emerald), pp. 163-188. DOI: 10.1108/S2041-806X (2012)0000009012.

López, A. y Benito, P. (2017) Prácticas económicas alternativas y resiliencia urbana: los mercados de productores y de trueque en Oviedo. Proyecto de investigación de Espacios y prácticas económicas alternativas para la construcción de la resiliencia de las ciudades españolas. CSO2015-65452-R (MINECO/FEDER).

Marx, K. (2017) El Capital, tomo I, Libro primero. El proceso de producción del capital. Zacatecas, México: Librerías Allende.

Más de Mx (2017) Conoce las monedas alternativas que circulan en México. Laboratorio de Conciencia Digital: https://masdemx.com/2017/02/monedas-alternativas-comunitarias-mexico/ (Última consulta, 20 de diciembre 2020).

Mazariegos Henkie, E. P. (2011) Economía solidaria una alternativa para el desarrollo comunitario en Aldea Xepiacul, la nueva Santa Catarina Ixtahuacán, Sololá. Tesis de Licenciatura, Universidad Rafael Landivar.Quetaltenango, Guatemala.

McLeay, M., Radia, A. y Thomas, R. (2015) LA CREACIÓN DE DINERO EN LA ECONOMÍA MODERNA. Revista de Economía Institucional, 17(33), pp. 355-383. [fecha de Consulta 3 de febrero de 2021]. ISSN: 0124-5996. Disponible en: https://www.redalyc.org/pdf/419/41943054016.pdf.

Méndez, J. (2004) Fundamentos de Economía. México: McGraw-Hill Interamericana.

Mishkin, F. (2014) Moneda, banca y mercados financieros. $10^{\circ}$ ed. Estado de México, México: Pearson Educación. Interamericana.

Nájar, A. (2012) El pueblo que invento sus billetes. BBC Mundo, Unidad de Información y Documentación de los Pueblos Indígenas del Noroeste de México y Hemerografía. Repositorio Institucional de la Biblioteca Gerardo $\begin{array}{llllll}\text { Cornejo } & \text { Murrieta } & \text { El Colegio } & \text { de }\end{array}$ 
https://www.bbc.com/mundo/noticias/2012/01/120126_tumin_dinero_mexico_an (Última consulta, 12 de diciembre 2020).

Ochoa Martínez, M. (2016) La Economía Solidaria como generadora de procesos de aprendizaje: el caso de la moneda comunitaria Túmin. Tesis Maestría, Universidad Veracruzana.

Orzi, R. (2017) ¿Otra moneda para otra economía? La necesidad de las monedas complementarias para el desarrollo sustentable de los emprendimientos de la economía social y solidaria. Revista del Departamento de Ciencias Sociales, 04, $\mathrm{N}^{\circ} 03$, pp. 155-171.

Peixoto, P. (2003) Moneda Social 2. Universidad Nacional General Sarmiento. Brasil: Veraz Ltda. Recuperado de https://www.economiasolidaria.org/sites/default/files/news_attachments/Laotraeconomia.pdf\#page=307.

Petri, I., Rana, O., y Cosmin, G. (2010) SLA as a Complementary Currency in Peer-2-Peer Markets. In: Altmann, J., Rana, O.F. (eds.) GECON 2010. LNCS, vol. 6296, pp. 141-152. Springer, Heidelberg.

Primavera, H. (2003) Moneda Social 1. Universidad Nacional General Sarmiento. Brasil: Veraz Ltda. Recuperado de https://www.economiasolidaria.org/sites/default/files/news_attachments/Laotraeconomia.pdf\#page=307.

Robles, P. (2013) El itacate, moneda alternativa. Reporte índigo: https://www.reporteindigo.com/reporte/el-itacatemoneda-alternativa/ (Última consulta, 20 de diciembre de 2020).

Rodas Carpizo, A. R. y Rodas Arroyo, C. (2011) Economía Básica. Editorial Limusa, ISBN 9786070504884.

Santana Echeagaray, M. E. (s.f) Monedas comunitarias en México y Argentina. Algunas comparaciones. Recuperado desde

https://socialcurrency.sciencesconf.org/conference/socialcurrency/pages/Monedas_comunitarias_en_MA_xico_y_A rgentina_SANTANA.pdf.

Secretaria del Medio Ambiente (SEDEMA) (2016) ¿Qué es el Mercado de Trueque?: https://sedema.cdmx.gob.mx/programas/programa/mercado-de-trueque (Última consulta, 25 de enero 2021).

Seyfang, G. (2004) Time Banks: rewarding community self-help in the inner city? Community Development Journal, 39, $\mathrm{N}^{\circ}$ 1, pp. 62-71. https://doi.org/10.1093/cdj/39.1.62.

Seyfang, G. y Longhurs, N. (2013) Growing green money? Mapping community currencies for sustainable development. Ecological Economics.

Schettino, M. (2015) Economía en un día. Ciudad de México, México: Paidós.

Smith, A. (1994) La riqueza de las naciones. Madrid, España: Alianza editorial. ISBN: 978- 84-206-5096-8.

Telesur (2012) El Túmin, una moneda alternativa: https://www.youtube.com/watch?v=XYdDS2yYCJ4\&feature=youtu.be (Última consulta 15 de enero 2021).

Vale de Multitrueque Kuni (2015) Vale Multitrueque Kuni. Hacia una sociedad resiliente y sana a través de la confianza: kunitq.wixsite.com/Kuni (Última consulta, 5 de enero 2021). 